\title{
Why Cycling in 2007 Was Faster than Being Driven in 2017 in Tianjin
}

\author{
John Zacharias ${ }^{1}$ and Qiang Sheng ${ }^{2}$ \\ 1. Laboratory for Urban Process Modelling and Applications, Peking University, Beijing 1000871, China \\ 2. School of Architecture and Design, Beijing Jiaotong University, Beijing 100044, China
}

\begin{abstract}
Changes in the road environment of Tianjin were intended to speed up the traffic system and shift bicyclists to the metro, bus and car. The metro system was greatly expanded, along with a modernized bus fleet. App-based taxi services were also introduced. In 2007, travellers to the central area were intercepted to determine the starting point of their trip and their travel mode. The most time-efficient trips in 2007 were by bicycle (61\%). These trips were re-enacted in 2017 using taxi and metro as it was no longer physically possible to replicate most of the original bicycle trips. Trips greater than $5 \mathrm{~km}$ in distance were somewhat faster by taxi than they were by bicycle, but overall, travel time by taxi was greater than by the bicycle for those same trips in 2007. A network analysis of road changes provides explanation why longer trips became more efficient while short trips became less efficient. Travel by metro alone was much longer than the other two methods, but the combination of app-based bicycles and metro would render this travel method the most efficient.
\end{abstract}

Key words: Transport efficiency, non-motorized transport, road infrastructure, taxi hailing service, bicycle, private car.

\section{Introduction}

Cities in China have undergone major change in the road transport system since the late 1990s, in particular to support rapid motorization. In less than 20 years, major cities largely replaced a transport system dominated by non-motorized modes to one with a diversity of modes, including the addition of private cars, metro, and various forms of shared mobility. The major guiding principles for the rebuilding of the road system include increasing the throughput of motorized vehicles and improving the efficiency of the whole system. A reduced role for non-motorized transport, in particular the bicycle, has accompanied these developments. This comprehensive transformation of urban road infrastructure is unprecedented in the motorization age. Evidence for the efficacy of various components of such new infrastructure is available, but there has been little effort to evaluate how such a transformed system works as a whole; in particular,

Corresponding author: John Zacharias, Chair Professor, research fields: transport, human behavior. how such a reformed road system fulfills the key goal of improved efficiency. As the rebuilding effort matures across the major, developed cities in China, the time is right to review the achievement and to consider directions for enhancement. For this examination, we turn to the case of Tianjin, which has undergone the transformations just described and for which we have prior data (2007) to allow a longitudinal analysis, which is the ideal way to measure the effectiveness of all of the transformations considered together.

The question posed in the following research is: How do the technological enhancements to the Tianjin transport system since 2007 contribute to transport system efficiency? The principal enhancements are the introduction of an arterial road network over the entire city, with interchanges and other speed-accelerating devices, together with the suppression of many at-grade road intersections. The connectivity of the non-motorized system has been reduced in an effort to eliminate conflict with motorized traffic.

The implementation of this system has also entailed the restructuring of local habitats, to reduce and 
rationalize the connections with the major road system and internalize other movement within residential compounds. In Tianjin, as in all major cities in China, the explosion of app-based taxi-hailing systems and various new technological and regulatory controls, have made the transport system increasingly complex while offering enhanced choice. At the same time as the road system was being "regularized", the metro also underwent a massive buildup. The metro system itself was designed to serve the built-up city and with a concentration on the core. In this way, Tianjin from the mid-2000s set out to make the whole of the city accessible by motor vehicles, while promoting a shift from the bicycle to the metro [1]. After 2000, many cities then engaged in rapid build-up of their metro systems, with 42 cities in China actively building underground metro systems in 2018.

Urban trips remain highly complex phenomena in cities, dependent on spatial and land use factors, the number, type and completeness of the various systems of transport available, as well as characteristics of each of them [2]. In China, cities have somewhat different trajectories with regard to car ownership, metro development and non-motorized transport due to macro-economic and local transport policies. Tianjin, in particular, retained its high share of non-motorized transport much longer than did other cities. In 2002, bicycle trips made up $52 \%$ of all trips over $500 \mathrm{~m}$ with substantially less in the other major cities around the same year including Beijing (30\%), Guangzhou (26\%), Shanghai (38\%) and Shenzhen (19\%) [3].

\subsection{Efficiency in Public Transport}

Efficiency and system utilities in general are key concepts in urban planning in China, although operationalization is typically complex and dependent on varied local conditions. It is broadly assumed that systems operating at higher speeds should deliver higher levels of efficiency, because in theory they can move more individuals through a given channel in given time, and also provide time savings to the travelers.

There are various measures of transport system efficiency, including time efficiency, accessibility, and planning efficiency or completeness and integration. Our concern is with the first of these, time efficiency, because this parameter can take a preponderant role in choice. Time is often represented in utility as the reciprocal of some measure of generalized travel time. Generalized travel time is composed of actual travel time and the degree of uncertainty about travel time [4]. Since most urban travel is time-constrained, predictability is very important in ensuring observance of a schedule. Time constraints, which naturally militate in favour of time economies, and time minimization in low productivity activity generally support this representation of travel utility from the user's perspective. Ultimately, urban residents' travel mode choices reflect personal utility [5].

At the urban scale, considerations of time-related utility are only part of the larger spatial plan, which itself has major consequences on the amount of energy consumed; in other words, the number of motor vehicles on the roads [6]. Travel by automobile might be explicitly favored in the planning [7] but also a claimed form of TOD (transport-oriented development) that favours the urban rail system according to some authors [8]. For others, this acclaimed TOD in China is simply a way to facilitate further motorization [9]. To the extent that such a node-network system is implemented as the main activity structure of the city, it might obviate the purpose of a single, integrated bicycle system, as existed widely in China prior to motorization.

Planning has favored automobile access in new development in China [7], which has meant the widening of many existing urban roads. These larger travel spaces have a thermal environment that is much less attractive to non-motorized travel [10]. In general, transport planners hold that higher speeds also allow for the possibility of greater throughput for a given channel, although this actually depends to a 
considerable extent on traffic density. To achieve this desired greater throughput, the plan is drawn for the higher speeds of motor vehicles, not for the speeds of non-motorized users.

In this study, we reproduce the trips reported at a random intercept study at four central locations [1]. In that study, the question was the likelihood of bicyclists, who dominated the transport system, switching to the metro, based on a utility function. The bus was eliminated from comparison since it was generally slower than the bicycle over the entire city within the Third Ring Road at that time. In 2017, the metro system has seen substantial growth, while the road environment has undergone transformation in favor of motorized transport. As a consequence, personal car transport has grown while the bicycle has declined. It is no longer possible to use a bicycle to execute many of the trips recorded in 2007 because of many physical barriers and the reconfiguration of the road system. It is widely held that the app-based taxi is the most time-efficient way to travel at present in Tianjin and consequently this mode has seen massive growth in numbers of vehicles and trips. The question is whether the public or app-based taxi can execute the trips of the original survey in more efficient manner to destination than the mode chosen in the earlier survey, the bicycle. At the same time, in consideration of an intensive build-up of the metro system to 8 operating lines, we ask whether the metro now offers a more time-efficient way to destination than those original bicycle-based trips.

\subsection{The Planned Shift to Motorized Modes}

In the early 2000s, the bicycle continued to dominate the transport system in Tianjin; for example, $52 \%$ of trips more than $500 \mathrm{~m}$ were by bicycle in 2002 [11]. The bicycle system could be considered complete in that all roads, including the three Ring Roads, were useable by bicycles.

The dominance of bicycles in many local streets led to motor vehicles following the pace and trajectory of the bicycles. Taxis were just $4 \%$ of the reported trips in the survey, in keeping with figures for Tianjin at that time. In the 2007 study, the bicycle out-performed the bus for same-distance travel on nearly all the city within the Third Ring Road, at the time the limit of the contiguous urban area. The bus generally had low and declining patronage. Mode shares changed dramatically from 2012, with non-motorized transport decreasing to a tiny proportion of its level before the year 2000 (Fig. 1). That decline accompanies a rapid rise in the number of privately owned cars. In 2015, there were 19.5 private cars per 100 persons in Tianjin [12], still well below the level in Beijing or Guangzhou. The fledgling metro system in 2007 offered $15 \%$ of bicyclists time savings of 15 minutes or more if they could ride to the nearest metro station and drop their bicycles there. The destinations - in our case, the intercept locations for our participants-were all metro stations so there was minimal egress time. Of course, the reality is that real egress time to destination is likely to approach access time, with consequent further reductions in the numbers of bicyclists who could benefit from time savings by including part of the trip on metro.

The metro was under rapid development with the stated intention on the part of the Tianjin authorities that there should be a considerable shift of bicyclists to the metro. It was widely held that bicycles slowed down the traffic system and introduced high levels of conflict. The metro system in 2017 consists of six lines within the Third Ring Road, for a total of 86 stations, including extensions beyond the Third Ring Road. When lines 5 and 6 come into operation soon, there will be additional 77 stations. Ridership increased considerably as lines were added in 2012. At the same time, the number of privately owned vehicles also increased much faster than the permanent population (Fig. 2). It is well recognized that this promotion of private car ownership was and is part of the government's promotion of consumerism. There is also popular support for the car mode in the context of a 


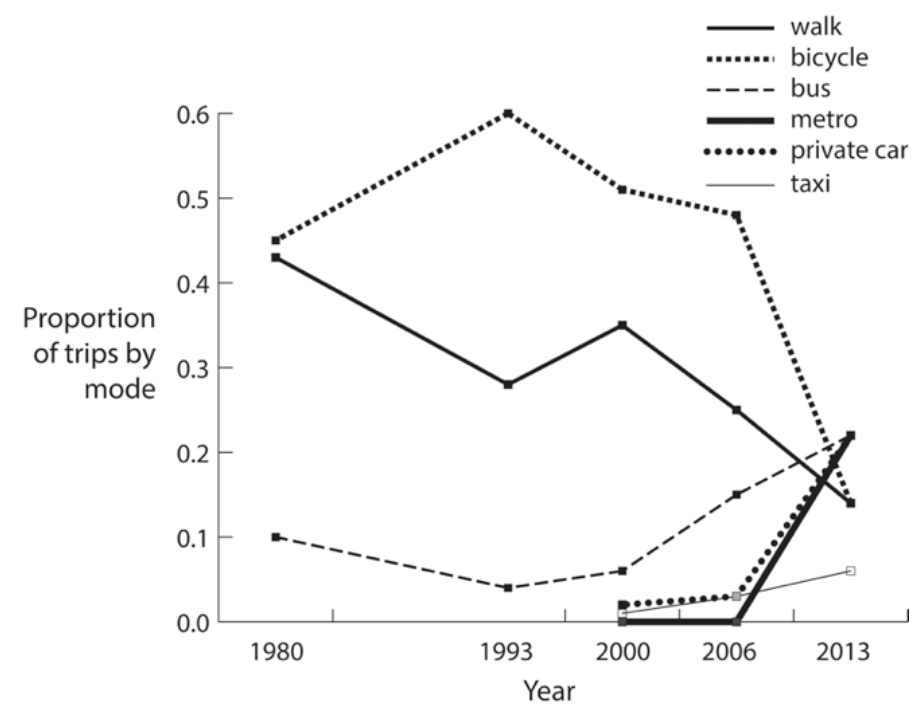

Fig. 1 The evolution in transport mode in Tianjin.

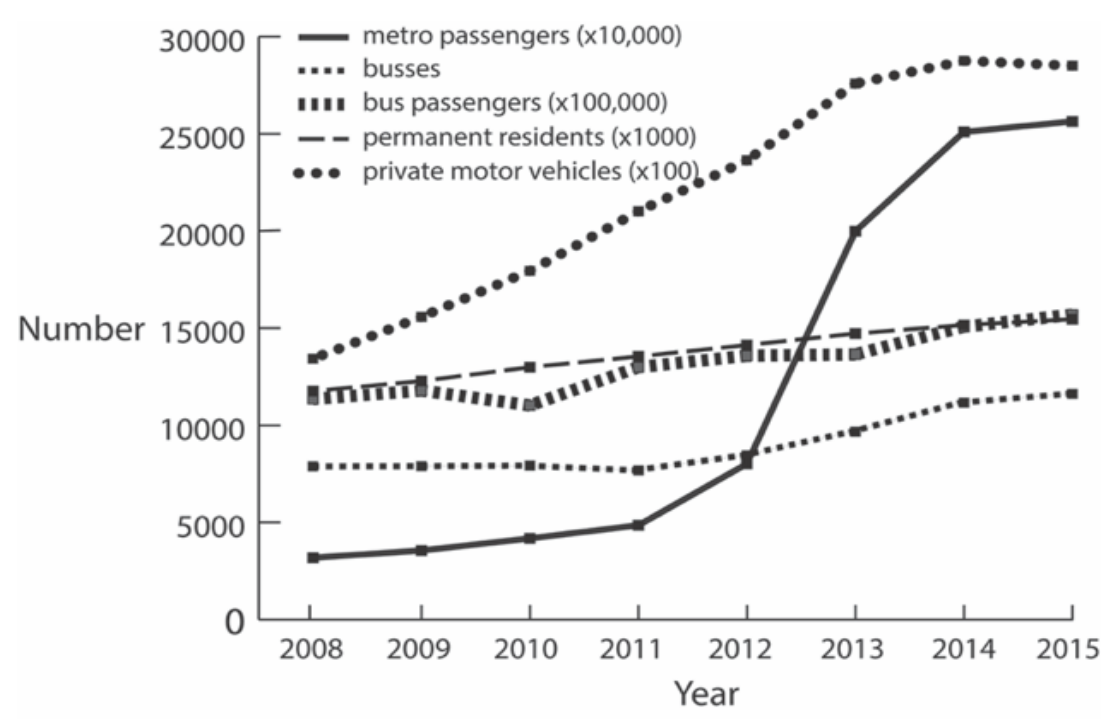

Fig. 2 Population, car ownership and metro evolution.

restructured city. In 2012, 88\% of young people surveyed in the Yangtze River Delta believed that a private car would enable them to travel faster, while $58 \%$ believed that having a car would be necessary in future [13]. In Tianjin a combination of disincentives to use the bicycle and major increases in road provisions for cars induced the demand for more car provisions.

\subsection{A Reformed Road System}

The basic layout of the Tianjin road system was preserved, while certain key roads were straightened and widened to make a complete, connected grid.
Previous roads that were largely undifferentiated and without barriers, consisted of two-way movement of vehicles and bicycles with no markings and with informal commercial or leisure activities curbside. While intersection controls had been introduced some years earlier, they were often ignored in 2007, when the first study was conducted. The roads were widened to 8 , 10 or 12 lanes, typically with three parallel fences, separating the bi-directional movement and non-motorized traffic from motor traffic. In this way, all turning traffic was restricted to the intersection. More light phases were introduced at level crossings to 
separate cross-movements. Many intersections saw the introduction of fly-overs or full-blown interchanges with continuous movement in all directions (Fig. 3). Bicycle routes were typically re-introduced in the liminal space of these new arterials and with crossings now incorporated with a reduced number of pedestrian crossings.

Fences to contain bicycle movement in the liminal space and to restrict left- and U-turns for vehicles were introduced after 2006. These fences were uninterrupted between major intersections, such that bicyclists could no longer use minor streets to travel directly to their destinations. They are now required to travel to the major intersection and wait for the green phase to proceed to the next major arterial. New residential habitat typically takes the form of a superblock that is often gated, with the two exits located at the mid-point along an arterial, in keeping with good engineering practice for the management of car traffic. Pedestrians and bicyclists must also use these exit locations from residential compounds, which entail a longer route to destination. These new habitats replace a highly connected street system that also connected directly to the major road infrastructure.

In the following, we analyze how the road system was reformed in the effort to promote the domination of faster-moving motor traffic. For this purpose, we use NAIN (normalized angular integration) [14], an advance on earlier methods that considered only topological distance [15]. NAIN measures the degree of fragmentation or integration of the road system-lower values reflect fragmentation while higher values integration. NAIN is expressed in the following way:

$$
N A I N \_r=\left(N C \_r\right)^{1.2} / A T D \_r
$$

where, NAIN_r denotes the normalized angular integration at metric radius of $r, A T D \_r$ indicates angular total depth at $r$. Angular total depth of each street segment measures the sum of angular distance from this street segment to all other segments within a certain radius. $N C_{-} r$ is the count of street segments within a given radius at node $r$.
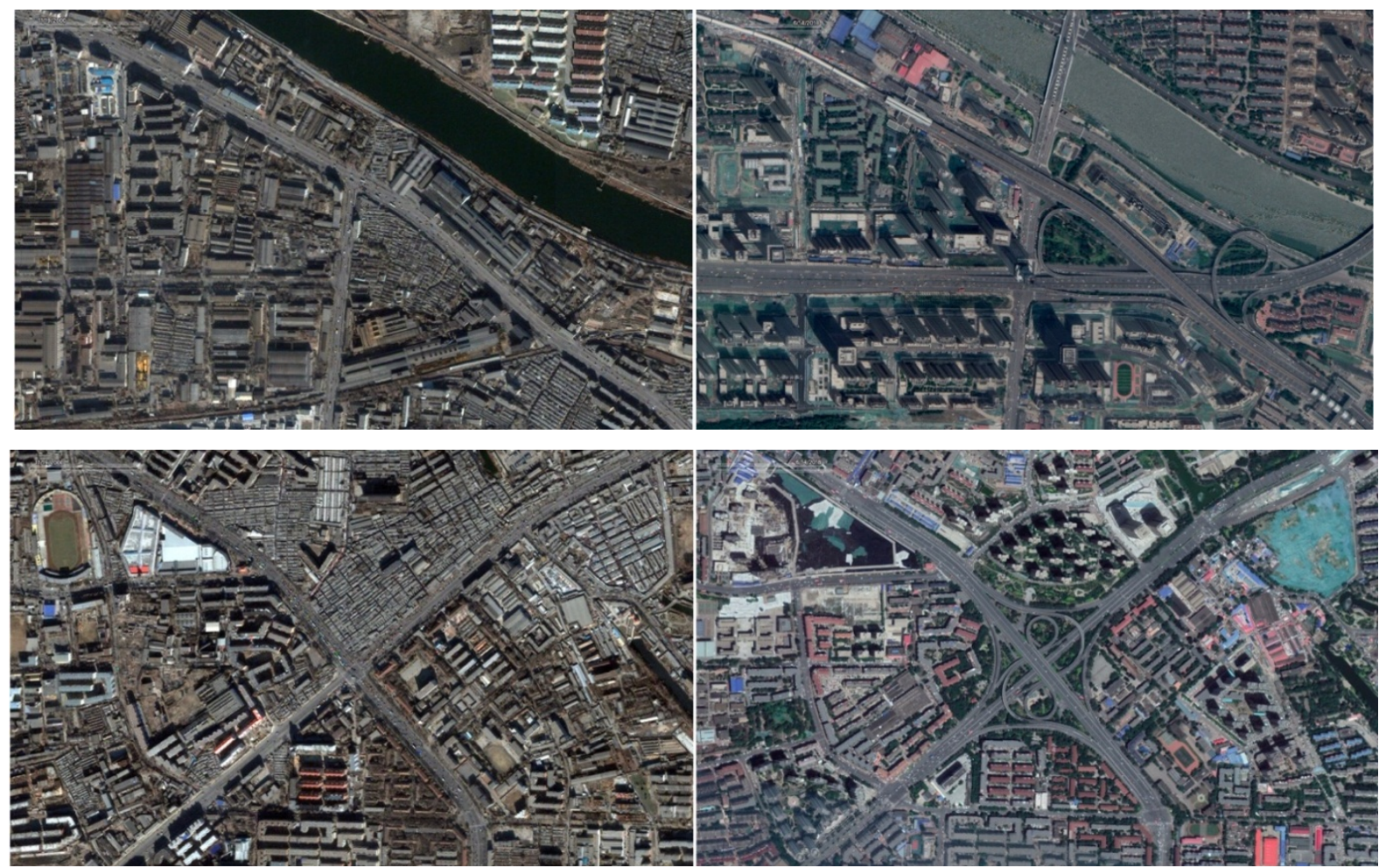

Fig. 3 Transformation in the road system in the effort to promote efficiency (c2000 left, c2017 right). 
In highly integrated road systems, one location is closer to another in topological terms, while more fragmented road systems require more circuitous travel, which creates greater topological distance, as well as greater metric distance in the majority of cases, between points.

Overall, at 2 to $5 \mathrm{~km}$ trip distance, there is a marked decline in integration of the road system in Tianjin between 2004 and 2017 (Fig. 4). In real terms, this means that distances between any two points at this
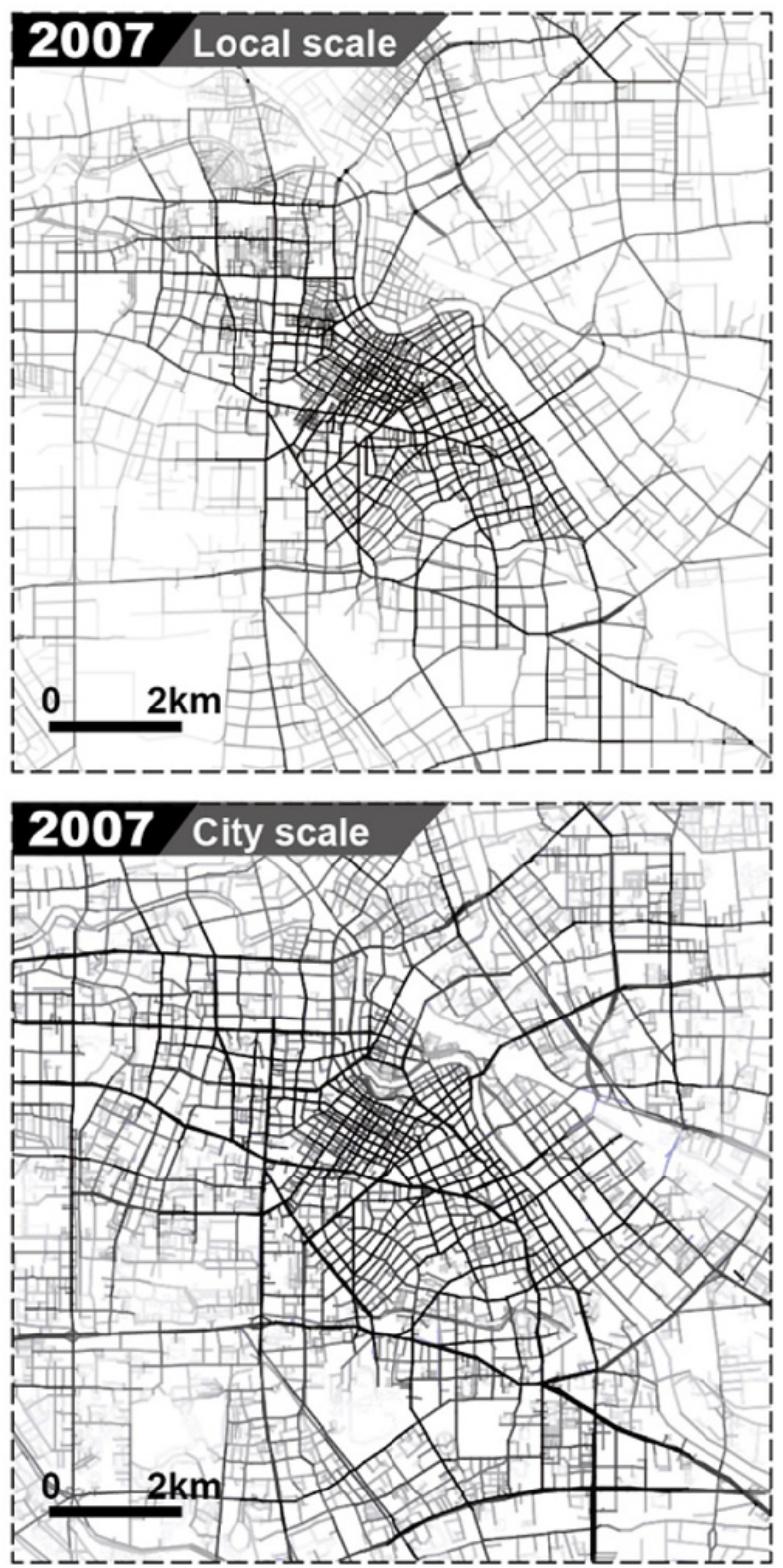

spatial scale increase. At the larger urban scale, there is greater integration, which presumably facilitates faster movement between any two points at this larger urban scale beyond $5 \mathrm{~km}$ radius from the origin point. Table 1 summarizes the results of this analysis. There is a decline in the maximum value of NAIN up to $5 \mathrm{~km}$, but average radii values are increasing. This is because there was a great deal of infill in the city in the intervening years and formerly small-scale urban fabric was replaced with large-scale modern grids. In effect,
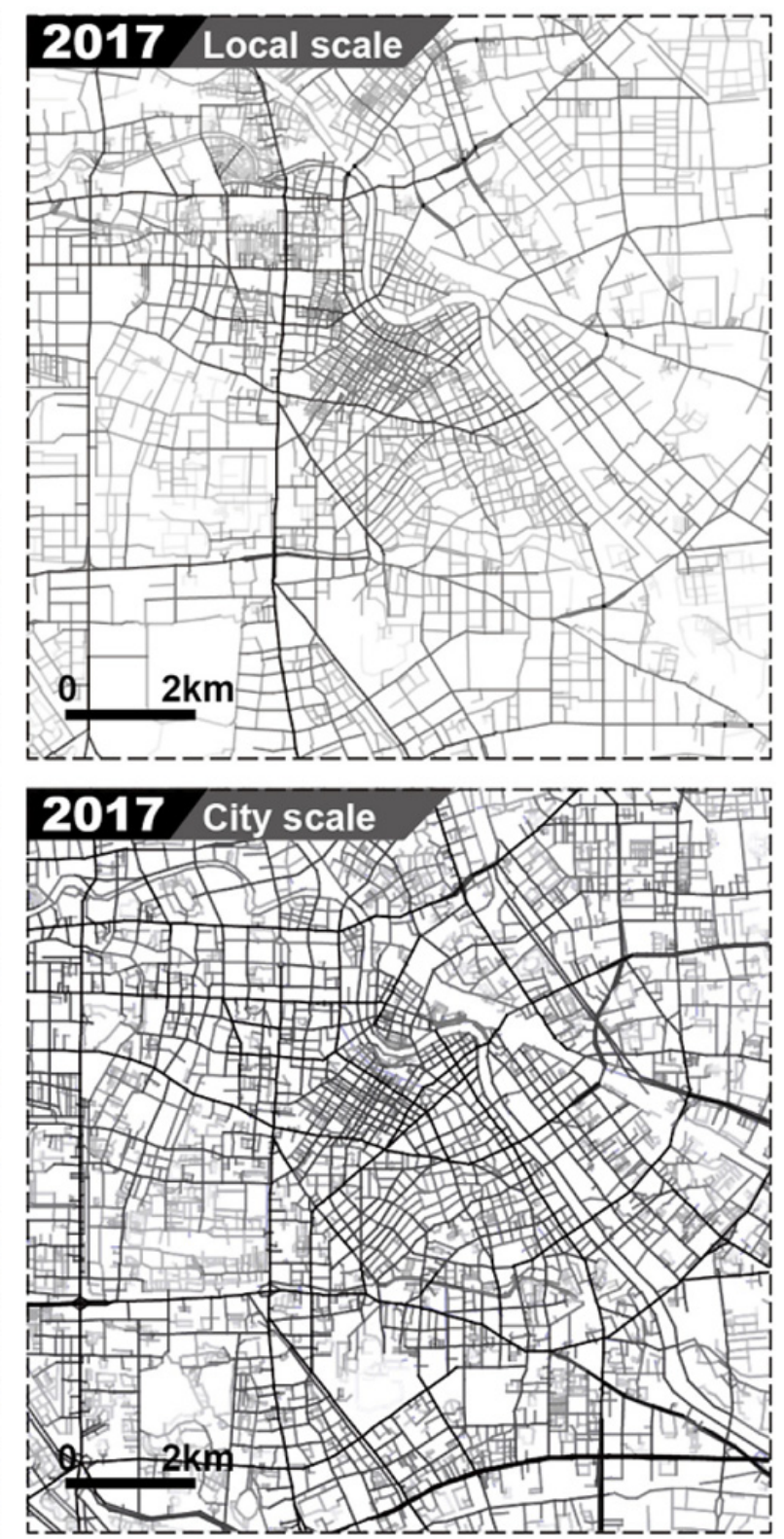

Fig. 4 The integration analysis of the road system of Tianjin in 2007 and 2017. Darker lines are more integrated. 
Table 1 Changes in NAIN values between 2004 and 2017 in Tianjin.

\begin{tabular}{lllllll}
\hline Spatial scale $(\mathrm{m})$ & \multicolumn{7}{c}{} \\
\cline { 2 - 6 } & 2,000 & 3,000 & 5,000 & 7,500 & 10,000 & 15,000 \\
\hline 2004 & & & & & \\
\hline Mean NAIN & 1.151 & 1.168 & 1.194 & 1.22 & 1.21 & 1.171 \\
Max NAIN & 2.232 & 2.186 & 2.084 & 2.002 & 1.896 & 1.700 \\
\hline 2017 & & & & & 1.323 & 1.321 \\
\hline Mean NAIN & 1.166 & 1.219 & 1.292 & 1.31 & 1.935 & 1.835 \\
Max NAIN & 2.196 & 2.055 & 2.034 & 1.968 & \\
\hline
\end{tabular}

transport planning traded less integration at the local scale for greater integration at the larger urban scale, which should have reduced travel time between points at greater urban distance. As will be seen later, travel speeds are indeed greater for those pairs of points at greater distance from each other, while points close in space result in much slower motor vehicle speeds. The question that this re-ordering of the road system in Tianjin raises is whether these efficiency measures do indeed improve on travel times overall, using the reputedly fastest means available, the app-based taxi. Secondly, we can investigate travel time between those same origin-destination pairs by a much expanded metro system, to see whether such trips offer time savings over the original bicycle trips.

\section{Methods and Materials}

The present study focuses on a single measure of overall efficiency - the time to execute a trip from a declared origin to a destination. Such time will be broken into parts in the case of the app-based car or the metro. There is the access time waiting for the responding car driver, or the walk to the metro entrance, either of which may constitute a major part of the total time. The main objective is to see the quantitative difference in the performance of the prevailing alternative transport systems between 2007 and 2017, in terms of time efficiency. The 2007 study was a pedestrian intercept with questionnaire at 4 central locations, all future metro station locations. For the 2017 data, an assistant went to the origin location and opened the taxi-hailing app. From this moment there is time until the assistant enters the motor vehicle. The trip in the car is recorded using a GPS emitter in real time (Codoon $®)$. Finally, the trips are also "executed" through the walk plus metro combination using an online app (http://www.tjgdjt.com). Times and distances were extracted for descriptive and correlation analyses. The main result of the study is the time efficiency of trips executed by bicycle and their equivalents by metro in 2007, and those same trips again in 2017.

In the 2007 study, two research assistants intercepted passersby, who provided their starting location before coming to the central location of the survey. Typically, the starting location was home. Smaller proportions of reported trips were by public bus, metro and on foot, than for reported bicycle trips. A shortest duration trip criterion was used to find the likely trajectory. Mean travel speed was determined by sampling executed trips. In the present study, the origin-destination pairs are now subject to new modes of transport: a much-increased metro system, and Didi, the app-based chauffeured car, and the regular taxi when available. The reworked layout clearly favors higher speeds as well as increased vehicular volume over a territory with much more even accessibility overall but where the bicycle now plays a minor role.

Estimated times for the most recent trip in 2007 were obtained from participants and the trace of their trip was verified with a GPS with travel time estimated from manual estimations from the field. For these parameters, averages were derived from multiple trips executed by walking, bicycling and bus. Metro times were obtained from the metro corporation. Taxi times were given by a local taxi company, with average 
operating speed at $28.8 \mathrm{~km} / \mathrm{h}$ for the trip duration.

\section{Results}

We assume that the time for departure is decided in advance, such that at imminent departure, the transport mode is immediately engaged. In the case of the bicycle, typically stored at the bottom of the access stairway in the residential block or in a yard adjacent to the building, the rider may need only a few moments to be in the saddle and cycling. In the case of app-based taxi hailing, we assume that equivalent imminent departure is engaging the app. Thus, there is a wait time for the taxi in those cases where a call was made. The period of hailing, confirming and waiting for the taxi, up to the point of embarkation, averaged 3.5 minutes, or $25 \%$ of the total time until the taxi arrived at destination. In $12 \%$ of the cases, eye contact with a taxi on the street initiated the use of the taxi, coded " 0 " for access time, although there is some time to identify any available taxis on the street, flag them and bring them roadside. These measures constitute a conservative estimate of the amount of time required for the above trips, which seems a good initial position for evaluating their usefulness to movement. Later enhancements could measure these transitional times that are typically discounted in travel time surveys.

By hailing app, the waiting time averaged $3.5 \mathrm{~min}$ and the total trip was $24.8(\mathrm{SD}=11.1)$. The trips by bicycle in 2007 averaged $19.8 \min (S D=19.3)$ at the same bicycle speed estimations for both periods. However, we know that the 2017 environment includes more delays and more detours for car-based travel, although long, unobstructed runs. We could also compare the two years and two modes by considering travel speed. With the wait time incorporated in the travel time, and with the use of the GIS, we obtain average travel speed, which is then adjusted for the variable waiting time. The travel speed for the taxi option was $4.14 \mathrm{~m} / \mathrm{s}(S D=1.57 ; n=392)$. The bicycle in the 2007 conditions would travel at $4.31 \mathrm{~m} / \mathrm{s}$ on average across the O-D pairs. The bicycle is faster than the app-based taxi or regular taxi.

It is well recognized that road-based traffic is subject to unpredictable delay due to congestion and incidents. Although there have been attempts at providing real-time information to drivers with regard to road conditions, such information is often based on small samples. For example, GPS-equipped taxis are used to estimate local travel speed, that is then converted into a real-time display of the road condition; however, travel speed may not be a good gauge of congestion conditions. In our study, 333 of 392 taxi trips followed the shortest distance trip, using the simple network representation of roads. Some of the remainder were longer than is represented by the network because of traffic prohibitions for left- and U-turns. A small proportion of trips were not distance-minimized because they opted to travel some of the distance on the peripheral highway system. It is apparent, in particular, that taxi-drivers did not rely on real-time traffic information as supplied by Baidu and the local government, to make itinerary decisions, but opted for the distance-minimized route.

Of the 392 taxi rides, 160 are faster than the bicycle option, which has relatively limited velocity range, for most conditions of riding and with typical city bicycles. However, this still means that well over half the taxi trips were slower than a bicycle had been. When access time is included, the car option in the form of the taxi becomes more time-efficient after $6 \mathrm{~km}$ of ride, or $48 \%$ of trips with destinations at the centre of the city. As suggested by the network analysis above, speeds increase beyond the $5 \mathrm{~km}$ range from origin. For example, mean speed for taxis with trips under $5 \mathrm{~km}$ distance is $3.9 \mathrm{~m} / \mathrm{s}$, but $4.9 \mathrm{~m} / \mathrm{s}$ from 5 to $8 \mathrm{~km}$ distance. Trips longer than $8 \mathrm{~km}$ have a mean speed of $5.8 \mathrm{~m} / \mathrm{s}$. As suggested above, it remains somewhat faster to hail a taxi for longer trips, that is those trips traversing more than half the diameter of the built-up city. There nevertheless remains considerable doubt that the trip will in fact be faster even if the averages suggest this is the case. Minimum measured travelling speed in a taxi 
is just 0.35 the maximum speed without any obvious pattern to the location of local congestion, according to our 2017 survey.

In this survey we also modeled travel time from the origin and using walk mode and metro. In this case, the mean trip time is 35.2 minutes, of which 26.4 minutes is access and egress time on foot. Such access walks average $1.1 \mathrm{~km}(S D=0.7)$. This walk distance is long in relation to planning standards and the findings of several empirical studies of metro access in China [16, 17]. The median access distance to a metro entrance in our case is $879 \mathrm{~m}$, which may explain why a substantial proportion of the residential environment of Tianjin does not have rapid access to the metro system.

However, the metro can also be seen as a healthy alternative. Considering the access times on foot, access time at local bicycle speeds is about one-third that of walk time. If a shared bicycle system is offered, there is still some access and delivery time, but access may be without any wait time. The total travel could be reduced to 18.8 minutes, which is faster than the bicycle or the taxi service for the majority of origin-destination pairs. Of course, this assumes a bicycle is immediately available at the residential location and can be deposited at destination. In this case, with the bicycle as the primary access mode, the metro becomes the most time-efficient means for the majority of trips.

The frequency distribution by time is also of interest; in particular, to know whether the time gains through modal shift occur at particular distances and times from destination areas. Efficiency improves as the taxi trip lengthens, such that trips over $6 \mathrm{~km}$ will on average, be more efficient than bicycles for this travel distance.

Although it is beyond the purposes of the present paper, we should also consider the time savings themselves and perhaps in relation to other aspects of travel experience. How important are $6 \mathrm{~min}$ time savings on average, when the saving requires a shift from one mode to another? How do we perceive and evaluate these times spent in transit? Are there other aspects of the systems that favor one over the other in the perceptions of users? We did not explore these issues, because we were focused on the primary measurement of system efficiency, measured as time. However, the previous study in Shanghai [18] revealed many of the reasons for the preference to use a bicycle, which included but were not limited to time economies. A similar result was seen in the large urban study also in Shanghai [19], where bicyclists generally found riding a bicycle to be comfortable, or at least more comfortable than the public transport alternatives.

\section{Discussion}

In the 2007 results, bus trips were significantly longer than bicycle trips, although only about $20 \%$ overall. The bus was used for as many short-distance trips as long. Given that just $5 \%$ of the participants walked, we could surmise that many proceeded to bicycle for relatively short distances. In 2017, bicycle use has declined dramatically while car use has increased dramatically. Occasionally severe congestion and reduced air quality have accompanied this increase. Through a complex interaction of road reconfiguration, higher motor traffic volumes and prescribed routing, the bicycle share has dropped to about $13 \%$.

The metro was hardly an option to the great majority of bicyclists in 2007, but in 2017, it represented a greater option, providing quicker access than the original bicycle for $41 \%$ of participants. If bicycle were available to the metro station, then the metro would perform better than other mode options. The difficulty is the provision of a feeder system for bicycles that could operate locally but generally over the entire city, as predicted by Mobike and ofo, the industry leaders in app-based bicycle services. The answer to the problem of redistribution of shared bicycles, by these operators, is to flood the city with shared bicycles. Otherwise, the original private bicycle provides the best service. Despite the efforts of these operators, bicycle use remains low in Tianjin.

Classical reasoning suggests such a rebuild of the 
entire transport system based on a different modal split and technologies will bring much increased efficiency. However, such supply-minded optimism is now tempered with the knowledge that such increased supply brings induced demand. If the city is built up for the car, then cars will tend to proliferate and be used. It might appear a solution to promote several infrastructure systems simultaneously as many Chinese cities are currently trying to do. However, the infrastructures themselves do not combine easily, which means that optimal networks for much travel cannot be realized. The assumption of a fault-free and balanced system serving all needs simultaneously is a big one. The question is whether such a system can ever be optimal, or whether our overall optimal system is really the combination of several sub-optimal solutions.

Tianjin was well known as a relatively compact city with a dense road network over its history. It was the first city in China to develop a comprehensive tram system, open in 1907, which was abandoned in the early 1970s in favour of a bus system. In the time span 2007 to 2015, the number of privately owned motor vehicles in Tianjin increased $151 \%$. Bus riders remained stagnant from 1992 to 2003, but then bus passengers doubled in the next period to 2015, as Tianjiners abandoned the bicycle. Metro patronage growth has been even more dramatic, ten times higher in 2015 than in 2007 , but still only $256 \mathrm{M}$ trips in the year. The rapid growth in public transport ridership, with the consequent decline in bicycle use, comes at the price of efficiency, however.

The rapid emergence of taxi-hailing apps has led to a rapid increase in the number of chauffeur-driven vehicles on the road in cities in China, leading to serious concerns about loads on the roads. While it is true that the hailed taxi reduces the demand for shortand long-term parking, vehicles circulate with at least part of their time unoccupied. They also wait roadside for potential customers, reducing road capacity and impeding other activities such as goods-loading, as well as bicycle and pedestrian movement.

The average taxi speed in the 2017 study was 17.2 $\mathrm{km} / \mathrm{h}$, compared with the average speed of $28.8 \mathrm{~km} / \mathrm{h}$ in 2007. The decline in speed is in part due to local congestion as suggested above, but also because level intersection crossings are now more complex, involving the separation of different directional movements and longer delay time for the greater number of controlled intersections. Although there has been a concerted attempt to create free-flow conditions at some major intersections, as shown in Fig. 3, the elimination of lighted intersection controls has not compensated for overall loss of efficiency.

The time differences between the original trips by bicycle in Tianjin and the re-enactment of the trip using a taxi and simulation of the same trip via metro reveal that the bicycle was and potentially still is the fastest surface mode for internal trips. We can safely generalize to say that the introduction of motor vehicles and the transport infrastructure that enabled it, have reduced the speeds and increased travel time for the general population across the city. Even in the context of a somewhat larger urban population and higher levels of mobility today, the new transport infrastructure does not address the basic needs of people to get to locations by minimizing time. Rather, it privileges certain modes - private cars and taxis as well as goods transport-by supplying capacity, in the process lowering the efficiency of the whole transport system.

Today, 8 lines of metro are built and the planned road system is mainly in place. The metro has not prevented the decline in car speeds from their 2007 levels. As a result, it is difficult to imagine that the present transport system can accommodate the further expansion of the car fleet-which now exceeds 2 $\mathrm{M}$-if not to see the reduction in its use. The local introduction of a shared bicycle, as presently operated, poses difficulties as a viable replacement for the walking trip access to the metro station for some trips.

There are several limitations to the present study. 
We have no estimate for how time savings might be traded for comfort or minimized effort. It would not be correct at this stage to infer likely modal shift based on time savings alone. The original itineraries were obtained from the 2007 participants, but travel time by bicycle was estimated from field trials. Cycling speed was based on averaged speeds of sample trips executed in 2007 in a road environment dominated by bicycles and adapted to them. In 2017, that kind of street environment had given way to a highly regulated and motorized road environment where bicycle speeds are likely lower overall than in 2007; however, we used the 2007 speed in the present comparison. We also did not execute the same trips by bicycle in 2017. Although in theory the trips could be executed, they rarely would have the same trajectory and involve more deviation from a straight line because of traffic regulations and barriers.

In the context of ongoing efforts to re-introduce non-motorized transport to Chinese cities-shared bicycle systems, efforts to build TOD - the issues are at more than one scale. In this study we looked at the impact of transportation system interventions on travel time for typical intra-urban trips. A further step would involve investigating the impact of large-scale concepts for the organization of non-motorized transport in the city.

\section{Acknowledgements}

The study was supported by the State Administration for Foreign Expert Affairs of China. Students at the University of Tianjin conducted the field study, while students at Peking University created the database.

\section{Conflict of Interest}

The authors declare no conflict of interest in the preparation of this manuscript.

\section{References}

[1] Zacharias, J., and Zhang, J. M. 2008. "Estimating the Shift from Bicycle to Metro in Tianjin." International Development Planning Review 30 (1): 93-111.
[2] Dai, D., Zhou, C., and Ye, C. 2016. "Spatial-temporal Characteristics and Factors Influencing Commuting Activities of Middle-class Residents in Guangzhou City, China." Chinese Geographical Science 26 (3): 410-28.

[3] Zacharias, J. 2003. "The Search for Sustainable Transport in a Developing City-The Case of Tianjin." International Development Planning Review 25 (3): 283-99.

[4] An, S., Leng, J.-Q., Wang, J. et al. 2015. "Vulnerability of Road Network Based on Generalised Travel Time." Proceedings of the Institution of Civil Engineers-Transport 168 (5): 425-33.

[5] Geng, J., Long, R., Chen, H. et al. 2017. "Exploration Multiple Motivations on Urban Residents' Travel Mode Choices: An Empirical Study from Jiangsu Province in China." Sustainability 9 (1): 136.

[6] Zhao, P., Diao, J., and Li, S. 2017. "The Influence of Urban Structure on Individual Transport Energy Consumption in China's Growing Cities." Habitat International 66: 95-105.

[7] Liu, X., and Zhou, J. 2014. "Spatial Pattern of Land Use and Its Implications for Mode-based Accessibility: Case Study of Nanjing, China." J. Urban Plan. Dev. 2014: 141.

[8] Dou, Y., Luo, X., Dong, L. et al. 2016. “An Empirical Study on Transit-oriented Low-carbon Urban Land Use Planning: Exploratory Spatial Data Analysis (ESDA) on Shanghai, China." Habitat International 53: 379-89.

[9] Doulet, J.-F., Delpirou, A., and Delaunay, T. 2017. "Taking Advantage of a Historic Opportunity? A Critical Review of the Literature on TOD in China." Journal of Transport and Land Use 10 (1): 77-92.

[10] Kuang, W. H., Yang, T. R., Liu, A. L., Zhang, C., Lu, D. S., and Chi, W. F. 2017. "An EcoCity Model for Regulation Urban Land Cover Structure and Thermal Environment: Taking Beijing as an Example." Science China: Earth Sciences 60 (6): 1098-109.

[11] Tianjin Statistical Yearbook. 2003. Tianjin: Tianjin Government.

https://www.chinayearbooks.com/tags/tianjin-statistical-y earbook.

[12] Collins, G., and Erickson, A. 2017. "Counting Cars: Rising Private Automobile Ownership in Chinese Cities Paves the Road for Gasoline Demand." China Signpost (2017). Accessed 2017-12-21. http://www.chinasignpost. com/2013/06/23/counting-cars-rising-private-automobileownership-in-chinese-cities-paves-road-for-gasoline-dem and/.

[13] Zhu, C., Zhu, Y., Lu, R., He, R., and Xia, Z. 2012. "Perceptions and Aspirations for Car Ownership among Chinese Students Attending Two Universities in the Yangtze Delta, China." Journal of Transport Geography 24: 315-25. 
[14] Hillier, B., Yang, T., and Turner, A. 2012. "Normalising Least Angle Choice in DepthMap and How It Opens up New Perspectives on the Global and Local Analysis of City Space.” Journal of Space Syntax 3: 155-93.

[15] Hillier, B., Penn, A., Hanson, J., Grajewski, T., and Xu, J. 1993. "Natural Movement: Or, Configuration and Attraction in Urban Pedestrian Movement." Environment and Planning B: Planning and Design 20: 29-66.

[16] Zacharias, J., and Zhao, Q. 2017. "Local Environmental Factors in Metro Patronage.” Journal of Public Transport.
DOI: 10.1007/s12469-017-0174-y.

[17] Sun, G., Zacharias, J., Ma, B., and Oreskovic, N. 2016. "Walking from Metro: The Effect of Local Built Environment in Beijing." Cities 56: 91-8.

[18] Zacharias, J. 2002. "Bicycle in Shanghai: Movement Patterns, Cyclist Attitudes and the Impact of Traffic Separation." Transport Reviews 22 (3): 309-22.

[19] Zacharias, J. 2005. "Non-motorized Transportation in Four Shanghai Districts." International Planning Studies 10 (3): $323-40$ 